\title{
Online Consumer Health Information: Awareness, Utilization and The Path to Self Medication
}

\author{
Parivash Badar* and Syed Mikhail Zaidi \\ Ziauddin University, Pakistan
}

*Corresponding author: Parivash Badar, Ziauddin University, Karachi, Pakistan

\author{
Received Date: May 27, 2019 \\ Published Date: May 29, 2019
}

\section{Introduction}

According to the World Health Organization, health has been defined as "a state of complete physical, mental and social wellbeing and not merely the absence of disease or infirmity" [1]. Health websites are an internet-based source providing information regarding all aspects of medicine. In recent years, with increasing numbers of people using the Internet, health websites have gained tremendous popularity. An article published in 2009 says, 51\% of adults in the ages of 18-64 in the US had used the Internet to look up health information [2]. An article published in the Health Informatics Journal that compared the use of information on the internet during 2000 and 2006 states that there has been a dramatic and significant increase in the number of people using using the internet for information regarding their health by $39 \%$ [3] According to an article, "more than 70,000 websites disseminate health information" with over "50 million people seeking health information online" [4]. People are making use of health websites for various reasons including academic, health awareness and general knowledge. Patients use health websites to learn more about their illnesses. A joint report released by the Council on Ethical and Judicial Affairs (CEJA) says that "Informational websites often provide a wide range of information including information related to physicians' practices, or information regarding certain medical conditions or specific treatment options" [5].

According to a research analysis conducted by the Pew and American Life Project Fully $80 \%$ of adult Internet users, have searched for at least one of 16 major health topics online [6]. People have access to causes and symptoms of different diseases, types of medicines, and other treatment plans. There are now websites that cater to specific diseases that have been setup by different sources such as patients or charities [7]. Furthermore, patients can find answers to the daily health issues they are facing such as side effects or complications of medical therapy [8].

The proportion of internet users in Pakistan is on a steep rise. During the years 2009 to 2013, 10 out of every 100 individuals, according to the World Bank [9], are internet users. Thus more people have access to health websites but no significant local research has been done regarding it. One study carried out in Pakistan focused only on Pakistani Health Websites found that the number of websites that provide health information and the number of people using and supporting them have increased in recent years [10] We felt that further exploration of this aspect of online consumer health information is becoming increasingly relevant. Donald Mackay wrote, "It includes information on specific illnesses and conditions, on good health and the prevention of ill health in general, and information to enable us to make informed choices about medical treatment" [11]. According to the CEJA report, "Health-related websites offer a wide range of information and services and are used by health professionals, patients, and the public with increasing frequency" [12]. With such convenient access the use of health websites, may have several disadvantages including self-medication, access to inaccurate information fall in the number of patient visits to physicians.

\section{Objectives}

A. To assess the pattern of usage of health websites among individuals.

B. To assess the frequency of usage of health websites in patients with long-term diseases.

C. To find out the frequency of self-medication in frequent users of health websites.

\section{Methodology}

We conducted a cross sectional study using a web based survey. We assessed the patterns of use of health websites usage among individuals. We also assessed the association of health website usage to the frequency of visits to different health websites in a year. The sample was selected by convenience sampling. A total of 512 samples were included in the study. The respondents answered 
a self-administered questionnaire on the Internet. Data collected was analyzed using SPSS 20 software. Participants who have used health websites more than 3 times in the past year, for any reason were included in the study. The questionnaire consists of __ number of questions. Questions regarding demographic variables, Internet accessibility, reasons for usage of health websites, perception of information of health websites, general opinion of health websites and abuse of health websites were asked. The questionnaire was in English. Consent was taken before the respondents fill the questionnaire.

\section{Result}

Table 1: A total of 512 respondents participated in the survey. Out of 512, 10 people did not give their consent so were excluded from the study.

\begin{tabular}{|c|c|c|c|c|c|}
\hline \multicolumn{2}{|c|}{ Gender } & \multirow{2}{*}{\multicolumn{2}{|c|}{ Age Group }} & \multirow{2}{*}{\multicolumn{2}{|c|}{ Education }} \\
\hline Total Number of Males & Total Number of Females & & & & \\
\hline \multirow{7}{*}{213 males } & \multirow{7}{*}{289 females } & \multirow{2}{*}{ 18-24 years } & \multirow{2}{*}{217 respondents } & Primary School & 3 \\
\hline & & & & High School & 118 \\
\hline & & \multirow{2}{*}{$25-34$ years } & \multirow{2}{*}{151 respondents } & College & 65 \\
\hline & & & & Bachelors & 189 \\
\hline & & 35- 44 years & 68 respondents & Masters and further & 127 \\
\hline & & 45-64 years & 57 respondents & & \\
\hline & & 65 years and onwards & 9 respondents & & \\
\hline
\end{tabular}

A total of 512 respondents participated in the survey. Out of 512,10 people did not give their consent so were excluded from the study. The demographic variables of the other 502 respondents are given in Table 1. All the respondents were asked about the usage of health websites. $43 \%(n=217)$ of the respondents reported that they do not use health websites for varying reasons such as having no need to do so. Out of the $57 \%(n=285)$ who used health websites, the majority (31\%) reported that they accessed health websites less than once a month. 95\% ( $n=271)$ of these respondents found health websites beneficial and $75 \%(n=214)$ of them said that they changed their health behavior after reading information on health websites.

From the 285 respondents who use health websites, 271 of them $(95 \%)$ found that their experience on the health website was helpful. The younger age groups were seen to access Health Websites more frequently than the older age groups with $26 \%$ $(n=131)$ aged between eighteen and twenty four years and $16 \%$ $(n=81)$ aged between twenty five and thirty four years. It is seen that people who have received a higher education were more likely to access health websites, $18.7 \%(n=94)$ having done their Bachelors and $16.9 \%(n=85)$ did their masters and further.

The main reason respondents were using health websites was for their personal health education (28\%). Figure 1. Some wished to learn more about certain diseases for the benefit of family members suffering from said diseases, others were students carrying out research for their own knowledge, and some just visited health websites for General reading (16\%). From the 502 consenting respondents, 412 of them were not suffering from a chronic disease. 90 respondents were suffereing from one of the below mentioned chronic diseases. Out of 90, 27(30\%) did not use health websites, whereas $63(70 \%)$ of the respondents did use them Figure 2. 90 respondents suffered from a chronic disease such as Hypertension (20\%), Diabetes (10\%), Asthma (12\%) and various other diseases. $78 \%(n=70)$ of these 90 respondents said that Health Websites helped them better understand their disease.

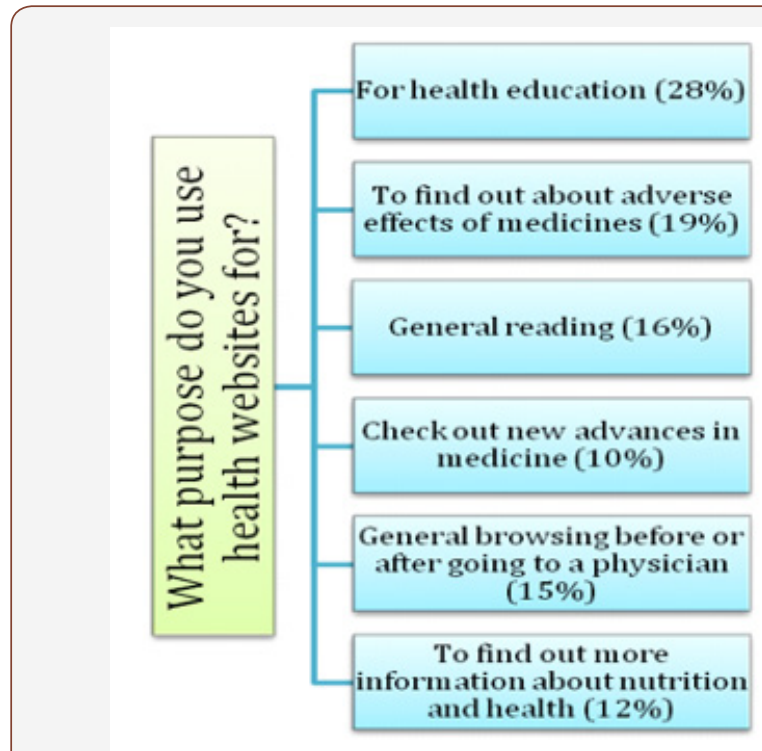

Figure 1: What purpose do you mainly use health websites for?

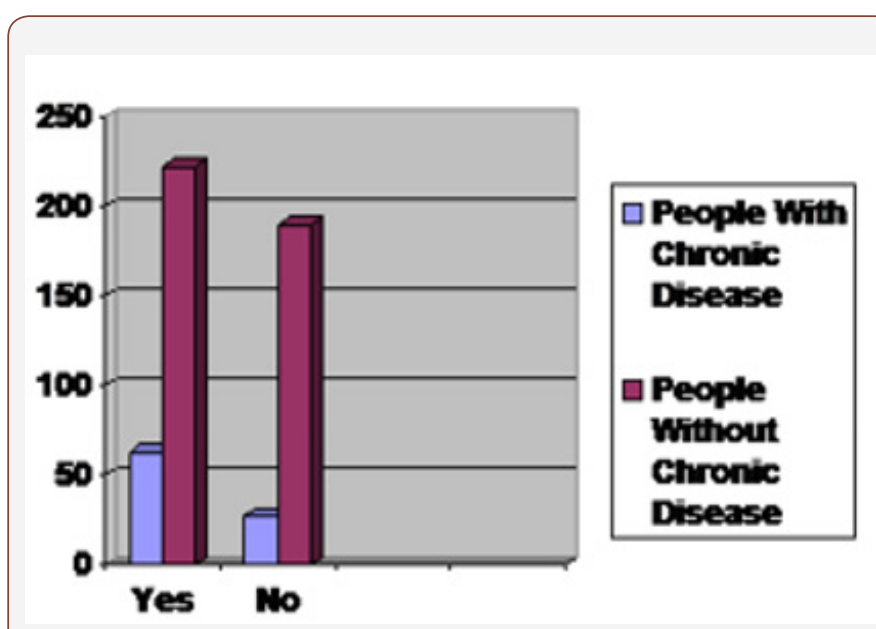

Figure 2: Usage of Health Websites amongst those with and without chronic disease. 
There was a significant proportion of people using health websites for self medication with the majority of them (75\%) doing so one to three times in the previous year Figure 3. Results were insignificant for different age groups. Out of the 502 respondents,
$59 \%(n=295)$ believe that people are more likely to resort to self medication after reading information on health websites. 357 (71\%) of the respondents said that they did not discontinue any drug after learning about its adverse effects on a health website.

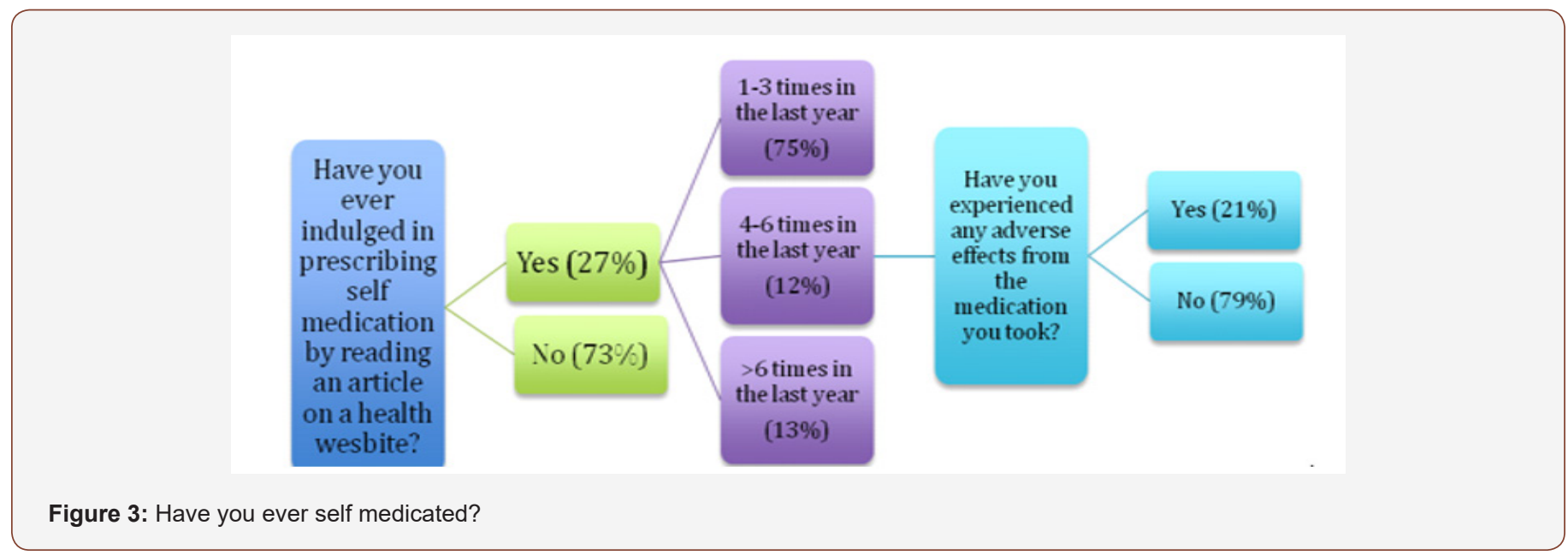

121 of the 502 (42\%) consenting respondents visited health websites before consulting a physician. Some of these experienced a change in their anxiety level whereas some felt no change at all. Out of those who visited health websites before visiting consultants, $71 \%(n=86)$ shared the information that they discovered online with their physicians. From these 25 respondents felt a decrease in anxiety level, 24 an increase in anxiety level, and 37 of the respondents felt no change at all.

\section{Discussion}

The use of health websites in a wide scope is distinctive [6]. It is a convenient tool that combines diverse pieces of information for specific and personal use and hence we see the trend of consumer use and demand to increase rapidly over a small amount of time [7, 9]. The focus of these websites is on the information structure that aims to empower consumers to manage their own health without the need to visit a licensed practitioner [9]. Its prime objective is therefore, to reduce the knowledge gap between a professional and a layman $[7,13]$. An overburdened practitioner and medical facilities now have the opportunity to link with other institution that can fill the roles of information providers for their patients [14, 15]. The invent of health websites during the past few years have led to an increase in social awareness amongst the masses and it is seen that people have started to take more interest in their health related issues [13]. People now have a wide access to hundreds of medical journals and experts advice from all over the world combined and put together in one single website as compared to the past where a licensed physician was their only source of knowledge [15]. This mass demand has led to the embellishment of these websites into various types: general health information provision sites, disease-specific sites, interactive patient group websites, scientific databases, and many other web tools [7]. However, the central focus of these websites is to serve a layman, they have progressively become more need-specific and hence allowing easy and convenient access to people [5].
Physician consultation time for a specific patient is calculated within about eight to eleven minutes on average due to the increasing burden on the doctors [16], this has lead to the sudden urge for more information by the patients [14]. The cost and time of visiting a licensed medical practitioner may seem unaffordable for many patients especially those in the rural areas of Pakistan, if they do not consider the illness or condition serious enough [17]. Managed care and other health providers see consumer health information as one tool to help improve patient satisfaction and reduce costs [18]. It also aims to support education, decision making, communication, and many other aspects of professional activity between the patient and the physician [13] It is easier to be situated at the comfort of your house and looking up certain condition and getting simple answer, convenience being the major advantage of health websites [9].

However, the information provided by these websites is not reviewed by an expert and lacks comprehensive coverage, some of these websites are not even updated regularly and thus fails to provide current information $[10,12]$. An ordinary man is likely to fall prey to misdiagnosis when he is made to believe that a particular condition is treatable when it is not, or a particular disease is not treatable when it in fact it is [9]. It is therefore often considered as misleading and outdated [11]. This is only a great advantage to the physician as he is protected from medical malpractice and incompetence in the future [7]. Regardless, of the large number of people resorting to health websites as an alternative there are still quite a few number of people who still consider their physicians the only reliable source and hence it is essential that their physicians should ask them about Internet use and counsel them about where to find reliable, accurate, high-quality health information and facilitating mutual support $[15,13]$.

The agencies marketing these websites have a few basic agendas upon which they hope to sell this product to its consumers [15] The major aim is to satisfy the need of a curious, ordinary 
man and hence, a sense of responsibility has been seen to emerge within the masses to take an active role in their healthcare $[18,15]$. The use of these websites have therefore, started to raise issues of 'consumerization' and 'responsibilisation' [7]. The question arises; Is an ordinary man cajoled into this? This has made very easy to label people as 'hypochondriacs' who affiliate themselves with signs and symptoms they may not even have just by reading the detailed reports present online [11]. These are some social and ethical issues that may be posing some detrimental personal issues within the consumers [19].

In Pakistan, obtaining drugs from a pharmacy without a prescription is quite easy. And hence the list of over the counter drugs is large and amendable according to ease. The only aspect holding people back is possibly the correct drug name. This is no longer difficult since health websites provide proper treatment plans with dosage and frequencies to their consumers. And hence, the biggest potential hazard imposed by the increasing use of health websites is the increasing trend of self-medication amongst the masses. The recreational use of certain medications such as sleeping pills and ADHD drugs by the youngsters has also led to an uprising of major social issues of dependence and liability amongst the youngsters.

WHO defines self-medication, as "medication that does not requires a medical prescription and which are produced, distributed and sold to consumers for use on their own initiative" [17]. Promotional messages through the internet sell medicines as just another consumer product however the patient may be mislead into buying a medicine that he/she may not have the full knowledge of [7]. A group of evaluation studies done to estimate the type of information wanted by the consumers stated that it appeared that the consumers did not know what to ask and how to ask it, spell it, abbreviate it or what is the logic or general to specific [3]. A layman reading pharmacokinetics and pharmacodynamics of a drug will not be able to comprehend the different interactions a drug is likely to have with other medications or the diet the patient may be taking, safety in pregnancy and lactation, use in children and the elderly, driving, working conditions compared to the more controlled prescription given out by the doctor [17] This failure to recognize contraindications, interactions, warnings and precautions may lead to the severe side effects and an increase in the levels of drug induced diseases in a community [18].

Health websites are definitely a very convenient and purposeful source of information, and they have played a great role in reducing the patient physician knowledge gap. They have allowed a layman to take an active role in regulating his personal health and thus reducing the burden of malpractice or incompetence on the medical practitioners. However, in the shadow of its ease the health websites have carved paths misdiagnosis and self-medication, increased over the counter drugs consumption and dependence and liability in the masses. These are some emerging threats that are detrimental to the masses, socially and personally.

\section{Conclusion}

With increased access to the Internet and online health information, there is a rise in the number of patients having acquired knowledge about their ailments, diseases and treatments. However, whether this knowledge is beneficial or harmful is still up for debate. Our research showed that $75 \%$ of the respondents found the use of Health Websites to be valuable.

\section{Acknowledgement}

None.

\section{Conflict of Interest}

No conflict of interest.

\section{References}

1. (2006) Constitution of the World Health Organization. Basic Documents, Forty-fifth edition.

2. Cohen RA, Stussman B (2005) Health information technology use among men and women aged 18-64: Early release of estimates from the National Health Interview Survey, January-June 2009. Health E-Stats National Center for Health Statistics.

3. O Bouhaddou, JG Lambert, S Miller (1998) Consumer health informatics: knowledge engineering and evaluation studies of medical HouseCall. Proc AMIA Symp 1998: 612-616.

4. Cline RJW, Haynes KM (2001) Consumer Health Information seeking on the internet: the state of the art. Health Education Research 16(6): 671692.

5. Morse LJ Council on Ethical and Judicial Affairs. Use of Health Related Websites. CEJA Report 6 - A-03.

6. (2003) Pew Internet \& American Life Project. Internet Health Resources. Health searches and email have become more commonplace, but there is room for improvement in searches and overall Internet access.

7. Personalized Health Care. Chapter 5.

8. Diaz JA, Griffith RA, Ng JJ, Reinert HYPERLINK SE, Friedman Hyperlink PD, et al. (2002) Patients' use of the Internet for medical information. J Gen Intern Med 17(3):180-185.

9. (2012) The World Bank. Data. Pakistan.

10. Mustafa K, Shah SA (2007) Online Consumer Health Information in Pakistan. PJLIS 8: 63-74.

11. Donald Mackay B\&W Chap 5.

12. Morse LJ Council on Ethical and Judicial Affairs. "Use of Health Related Websites". CEJA Report 6 - A-03

13. Eysenbach G (2000) Consumer health informatics. BMJ 320(7251): 1713-1716.

14. Deering MJ, Harris J (1996) Consumer health information demand and delivery: implications for libraries. Bull Med Libr Assoc 84(2): 209-216.

15. O Bouhaddou, JG Lambert, S Miller (1998) Consumer health informatics: knowledge engineering and evaluation studies of medical HouseCall. Proc AMIA Symp 1998: 612-616.

16. Fox S (2008) Pew Internet \& American Life Project. The engaged e-patient population.

17. (2000) World Health Organization. WHO Drug Information Pp. 4(1).

18. Deering MJ, Harris J (1996) Consumer health information demand and delivery: implications for libraries. Bull Med Libr Assoc 84(2): 209-216.

19. Cooley DL, Mancuso AM, Weiss LB, Coren JS (2011) Health-Related Internet Use Among Patients of Osteopathic Physicians. J Am Osteopath Assoc 111(8): 473-482.

20. World Health Organization. Guidelines for the Regulatory Assessment of Medicinal Products for Use in Self-Medication. Characteristics of Self Medication.

21. Eysenbach G (2000) Consumer health informatics. BMJ 320(7251): 1713-1716. 